\title{
Amisulpride - An Athypical Antipsychotic?
}

\author{
Echim George ${ }^{2}$, Sorina Cucuiet ${ }^{3}$, Bianca Osz ${ }^{1}$, Alexandra Grosan, Gal Zsolt ${ }^{1,2}$, Maria Dogaru ${ }^{1}$ \\ 1 Department of Pharmacology and Clinical Pharmacy, University of Medicine and Pharmacy of Tîrgu Mureș, Romania \\ 2 Doctoral School, University of Medicine and Pharmacy of Tîrgu Mureș, Romania \\ ${ }^{3}$ Department of Pharmacology, Faculty of Medicine, University of Medicine and Pharmacy of Tîrgu Mureș, Romania \\ 4 Pharmacy of The University of Tîrgu Mureș, Romania
}

\begin{abstract}
Aim: the purpose of the present study was to determine the effects of amisulpride at different doses on body weight, glucidic and lipidic metabolism.

Material and method: Thirty-six white Wistar rats were treated daily for 9 weeks with amisulpride $1 \mathrm{mg} / \mathrm{kg}$ and $10 \mathrm{mg} / \mathrm{kg}$ body weight. Another group received distilled water and served as control group. At the end of the treatment period blood samples were collected and the follow biochemical parameters were determined: serum cholesterol, triglycerides, blood glucose, GOT, GPT. Body weight gain was also assessed weekly.

Results: After treatment with amisulpride in doses of $1 \mathrm{mg} / \mathrm{kg}$ and $10 \mathrm{mg} / \mathrm{kg}$ for a period of nine weeks, weight gains were recorded for both groups compared with the initial weight and the control group. Blood glucose concentrations in the group treated with $1 \mathrm{mg}$ amisulpride/kg body weight were significantly increased ( $p<0.05$ vs control group), but in the group treated with $10 \mathrm{mg} / \mathrm{kg}$ body weight glucose levels were not statistically significant increased compared to controls. Other biochemical parameters (cholesterol, triglycerides, GOT, GPT) showed no statistically significant differences compared to control group.

Conclusions: amisulpride administered over a period of 9 weeks, in doses of $1 \mathrm{mg} / \mathrm{kg}$ and $10 \mathrm{mg} / \mathrm{kg}$ showed a slight increase of body weight regardless of gender, increased blood glucose only when was administered in the low dose, and does not affect lipid metabolism, even though decreased cholesterol and triglycerides levels. This results highlight a real benefit of treatment with amisulpride, comparatively with other athypical antipsychotics.
\end{abstract}

Keywords: amisulpride, lipid metabolism, glucidic metabolism, body weight.

Received: 24 June 2014 / Accepted: 31 July 2015

\section{Introduction}

Amisulpride is an athypical antipsychotic, used in the treatment of schizophrenia. It is a benzamide derivative, with antipsychotic and antidepressants properties, which mainly acts as specific antagonist of D2 receptors [1]. In literature there are controversial data regarding its pharmacological and pharmacotoxicological profile, namely its effects on body weight, its likelihood to induce lipid metabolism disturbances and about the risk of producing diabetes on long term administration $[2,3]$. The objective of our research is to perform a thoroughgoing study regarding the influence of amisulpride treatment in different doses for a 9 weeks period in Wistar rats on body weight and its risk to produce diabetes.

\section{Material and Methods}

In our study we used 36 white Wistar rats, male and female, assigned in 3 experimental groups.

Experimental protocol: Group no.1, consisting in 12 white Wistar rats (6 males and 6 females) received amisulpride $1 \mathrm{mg} / \mathrm{kg}$ body weight, and group no. 2, consisting in 12 white Wistar rats (6 males and 6 females) received amisulpride $10 \mathrm{mg} / \mathrm{kg}$ body weight, No.3 group consisting in 12 white Wistar rats, 6 males and 6 females received only distilled water without medication, for 9 weeks and

* Correspondence to: Sorina Cucuiet

E-mail: cucuiet.sorina@umftgm.ro served as control. For the whole study time the animals were groomed in UMF Biobasis, fed ad libitum with fodder and water, providing optimal temperature $22 \pm 2$ degrees Celsius and 30-60\% humidity. The animals were observed daily after gavage, at 1 hour, 2 hours and 4 hours to distinguish the side effects of the medication and were weighed weekly. All experimental procedures followed international guides regarding laboratory animals handling, considering the Directive 2010/63/EU which regulates the protection of animals used for scientific purposes and also with the approval of The Ethics Committee of The University of Medicine and Pharmacy Tîrgu Mureș. After 9 weeks of treatment the animals were weighed for recording the final body weight then were slaughtered (euthanasia with sodium phenobarbital i.v., with rigor mortis confirmation). Blood samples were taken for assessing the following biochemical parameters: serum cholesterol, triglycerides, GOT, GPT values and blood glucose. Biochemical parameters were measured using ABBOTT photometer tests.

\section{Statistical analysis}

All data are reported as mean \pm SD. The statistical analysis was performed using Student's t test. Statistical significance limit was set at $\mathrm{p}<0.05$.

\section{Results}

Weight gain after administration of a dose of $1 \mathrm{mg}$ amisulpride $/ \mathrm{kg}$ was assessed separately on female and male rats, 
in order to see if there are differences in weight gain regarding gender. The weight gain was calculated considering the entire group (female and male rats). (Figure 1-3)

As can be noticed above, amisulpride produced weight gain, both on female and male rats, without statistically significant differences between gender.

Assessing the weight gain after administration of amisulpride in dose of $10 \mathrm{mg} / \mathrm{kg}$ body weight. (Figure 4)

The weight gain recorded in the group receiving $10 \mathrm{mg} /$ $\mathrm{kg}$ body weight is less compared with the group receiving $1 \mathrm{mg} / \mathrm{kg}$ body weight, and likewise in the previous experiment there were no statistically significant differences between male and female rats.

As shown above there ares no statisticaly significant differences between intial and final weight.

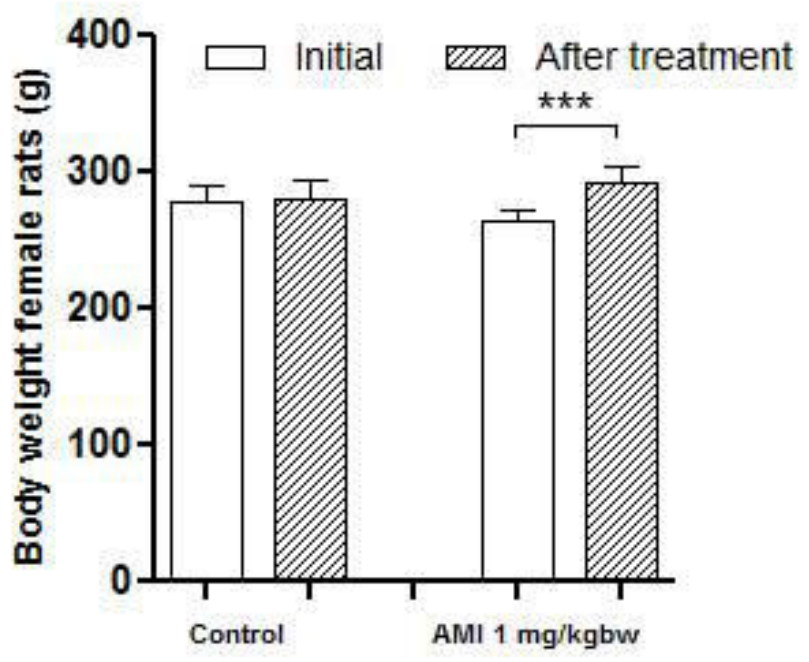

${ }^{* \star *}$ - $\mathrm{p}<0.05, \mathrm{P}$ values were calculated with Student $\mathrm{t}$-test; AMI - amisulpride

Fig. 1. Body weight determined before and after treatment with amisulpride for female rats (control group and amisulpride $1 \mathrm{mg} /$ $\mathrm{kg})$

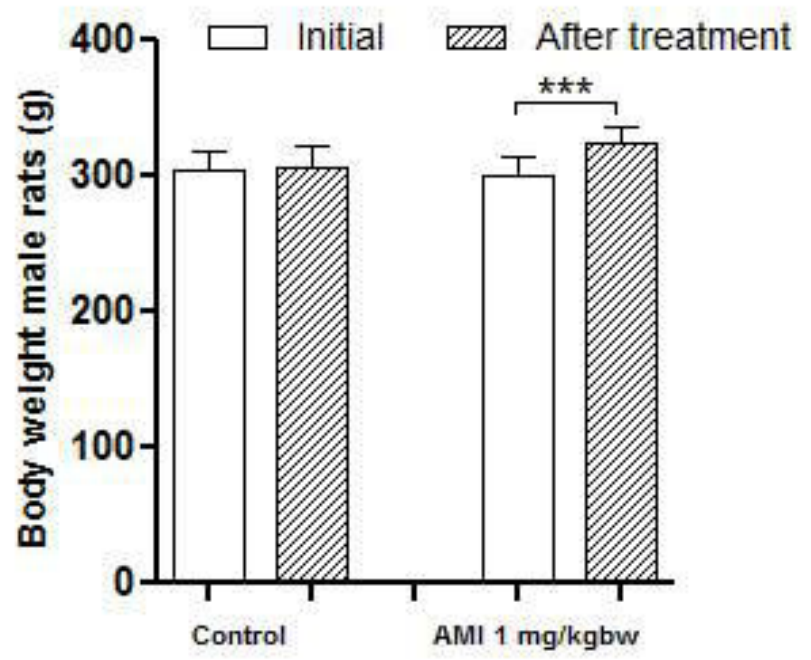

${ }^{* \star *}$ - $p<0.05, P$ values were calculated with Student $t$-test; AMI - amisulpride

Fig. 2.Body weight determined before and after treatment with amisulpride for male rats (control group and amisulpride $1 \mathrm{mg} / \mathrm{kg}$ )
The assessement of blood glucose before and after treatment with amisulpride $1 \mathrm{mg} / \mathrm{kg}$ bw comparative with control group. (Figure 5)

The assessment of blood glucose before and after treatment with amisulpride $10 \mathrm{mg} / \mathrm{kg}$ comparative with control group. (Figure 6)

The assessment of cholesterol and triglyceride levels after administration of amisulpride (Figure 7).

Interestingly, amisulpride in a dose of $1 \mathrm{mg} / \mathrm{kg}$ decreased total serum cholesterol with approximately $10 \%$ compared to control group $(\mathrm{p}<0.05)$ and serum triglyceride concentrations with $25 \%$ compared to control group $(\mathrm{p}<0.001)$.

We found that serum cholesterol level of rats treated with amisulpride $10 \mathrm{mg} / \mathrm{kg}$ compared to the control group showed no statisticaly significant difference $(\mathrm{p}>0.05$, $\mathrm{p}=0.2091$ ).

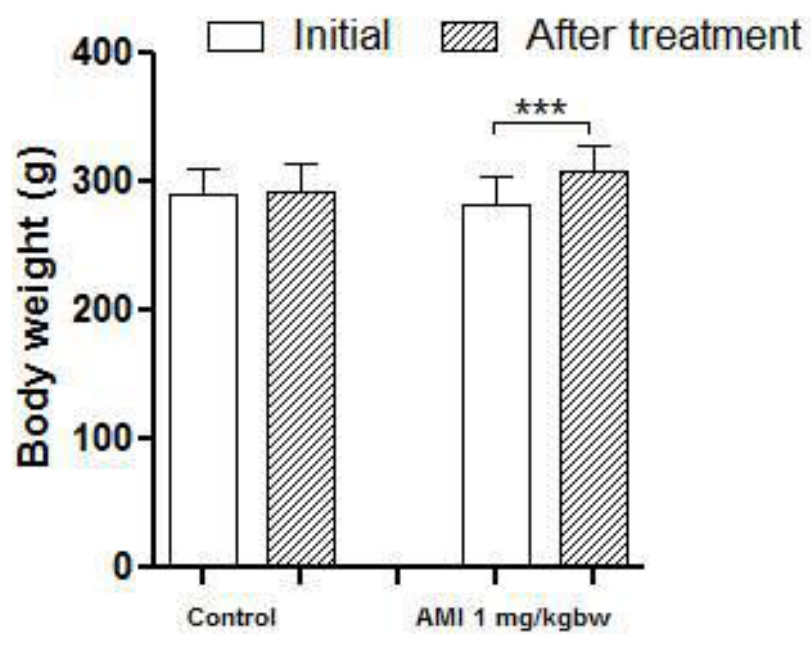

***- $\mathrm{p}<0.05, \mathrm{P}$ values were calculated with Student $\mathrm{t}$-test; AMI - amisulpride

Fig. 3. Body weight determined before and after treatment with amisulpride for entire group (male and female rats) (control group and amisulpride $1 \mathrm{mg} / \mathrm{kg}$ )

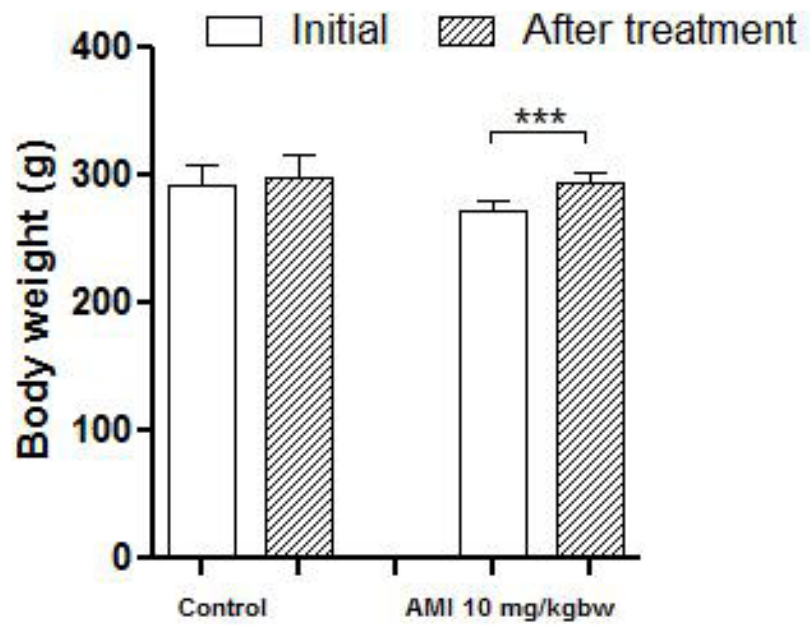

${ }^{* \star \star}$ - $\mathrm{p}<0.05, \mathrm{P}$ values were calculated with Student t-test; AMI - amisulpride

Fig. 4. Body weight determined before and after treatment with amisulpride (control group and amisulpride $10 \mathrm{mg} / \mathrm{kg}$ ) 
Serum triglyceride concentrations of rats treated with amisulpride $1 \mathrm{mg} / \mathrm{kg}$ and amisulpride $10 \mathrm{mg} / \mathrm{kg}$ compared to the control group. (Figure 8)

There is no statisticaly significant difference between group receiving amisulpride $10 \mathrm{mg} / \mathrm{kg}$ and control group $(\mathrm{p}>0.05)$.

The assessment of serum glutamic oxaloacetic transaminase (GOT) concentrations of rats treated with amisul-

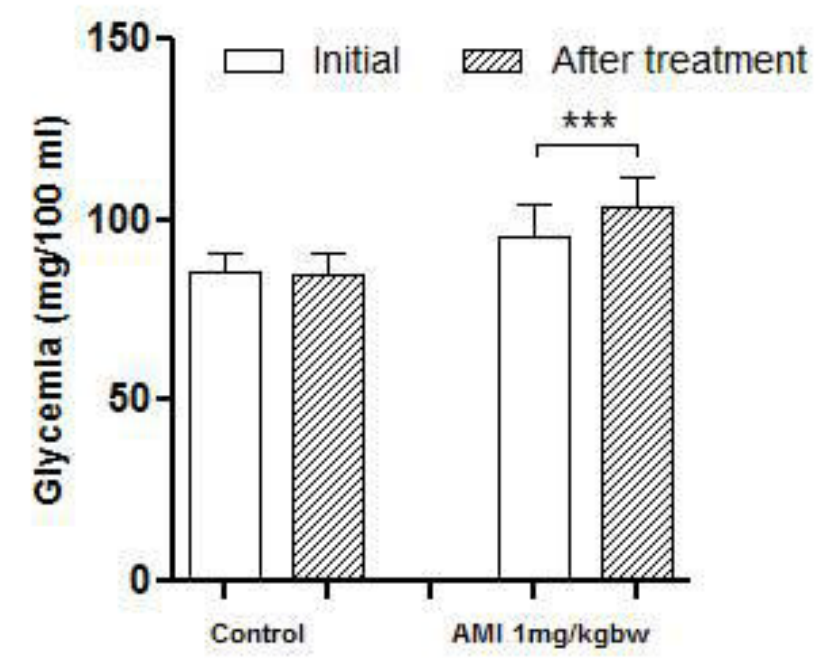

***- $\mathrm{p}<0.05$. P values were calculated with Student $\mathrm{t}$-test; AMI - amisulpride

Fig. 5. Glycemia determined before and after treatment with amisulpride (control group and amisulpride $1 \mathrm{mg} / \mathrm{kg}$ )

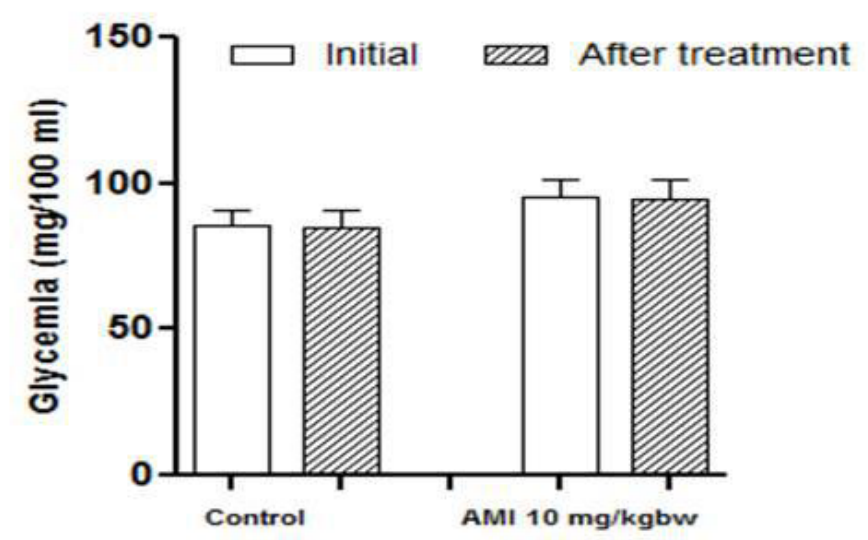

Fig. 6. Glycemia determined before and after treatment with amisulpride (control group and amisulpride $10 \mathrm{mg} / \mathrm{kg}$ ) ( $p>0.05$ )
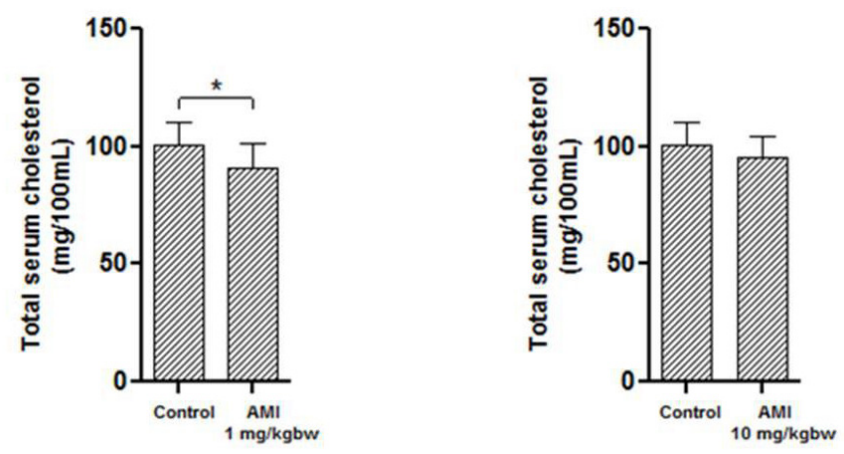

*- $p<0.05, P$ values were calculated with Student t-test; AMI - amisulprid

Fig. 7. Total serum cholesterol level of rats treated with amisulpride $1 \mathrm{mg} / \mathrm{kg}$ and amisulpride $10 \mathrm{mg} / \mathrm{kg}$ compared to the control group pride $1 \mathrm{mg} / \mathrm{kg}$ and amisulpride $10 \mathrm{mg} / \mathrm{kg}$ compared to the control group. (Figure 9)

The results showed that there is no statisticaly significant difference between group receiving amisulpride $1 \mathrm{mg} / \mathrm{kg}$ and conrol group ( $p>0.05)$, and also between group receiving amisulpride $10 \mathrm{mg} / \mathrm{kg}$ and control group ( $\mathrm{p}>0.05)$.

Serum glutamic-pyruvic transaminase (GPT) concentrations of rats treated with amisulpride $1 \mathrm{mg} / \mathrm{kg}$ and amisulpride $10 \mathrm{mg} / \mathrm{kg}$ compared to the control group. (Figure 10.)

As shown in Figure 10, serum glutamic-pyruvic transaminase (GPT) concentrations in rats receiving amisulpride $1 \mathrm{mg} / \mathrm{kg}$ were elevated compared to control group $(\mathrm{p}<0.005)$.


${ }^{* \star *}$ - $\mathrm{p}<0.05, \mathrm{P}$ values were calculated with Student t-test; AMI - amisulpride

Fig. 8. Serum triglyceride concentrations of rats treated with amisulpride $10 \mathrm{mg} / \mathrm{kg}$ compared to the control group


Fig. 9. Serum glutamic oxaloacetic transaminase (GOT) concentrations of rats treated with amisulpride $1 \mathrm{mg} / \mathrm{kg}$ and $10 \mathrm{mg} / \mathrm{kg}$ compared to the control group
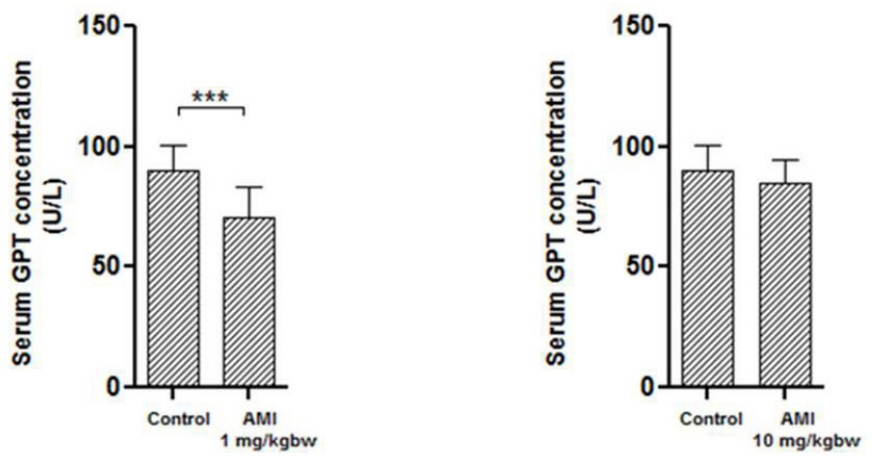

${ }^{\star \star \star}$ - $p<0.005$, P values were calculated with Student t-test; AMI - amisulpride.

Fig.10. Serum glutamic-pyruvic transaminase (GPT) concentrations of rats treated with amisulpride $1 \mathrm{mg} / \mathrm{kg}$ and $10 \mathrm{mg} / \mathrm{kg}$ compared to the control group 
Regarding serum glutamic-pyruvic transaminase (GPT) concentrations of rats treated with amisulpride $10 \mathrm{mg} / \mathrm{kg}$ compared to the control group, we found no statisticaly significant difference $(\mathrm{p}>0.05)$.

\section{Discussions}

The second-generation antipsychotics are associated with metabolic side effects such weight gain, increased body fat deposition, lipid metabolism disturbances and diabetes mellitus. These adverse effects are not only the risk factors for cardiovascular disease, insulin resistance and diabetes mellitus leading to increased morbidity and mortality, they may also impair the patient's adherence to treatment. The increased fat is known to cause resistance to insulin action. It is well known that the normal response to insulin resistance is compensatory hyperinsulinemia, which may prevent diabetes. In animals, there are evidence that the treatement with atypical antipsychotics cause an inadequate hyperinsulinemic compensation. Clozapine and olanzapine are showing the highest risk of producing significant weight gain, whereas aripiprazole and amisulpride are considered to be less prone to cause weight gain [4]. Studies performed in rodents and human volunteers have shown that atypical antipsychotics stimulate differentiation and maturation of preadipocytes in adipocytes, increasing adipose tissue, particularly in visceral area. The treatment with antipsychotics leads to decreased expression of the gene coding for the synthesis of glucose transporter GLUT4 in adipose tissue. The blockade of D2 receptors interfere with the activity of protein kinase $\mathrm{B}$, preventing activation through phosphorylation of the transporter GLUT 4. The insulin resistance observed after administration of antipsychotics is due to an increased secretion of leptin, a peptide hormone that regulates appetite and is produced by subcutaneous adipocytes, and due to development of relative or absolute leptin resistance. Fasting morning ghrelin levels decrease early in the course of antipsychotic treatment and then increase after chronic exposure. In addition to leptin and ghrelin, other hormones and receptors that have been associated with food intake and energy homeostasis are potentially involved in antipsychotic-induced weight gain [5]. There are also data demonstrating a high level of pro-inflammatory mediators in subjects with obesity and insulin resistance [2].

The blockade of D2 receptors potentiates the effects on food intake produced by blocking of 5HT2 receptors, produces disinhibition effect on prolactine secretion and has negative influence on glucidic and lipidic metabolism. The blockade of D2 receptors in reward system produces dysphoria and has as a consequence an increase in food intake. Antipsychotics that acts preferentially by blocking D2 receptors have not marked effects on body weight, but those acts preferentially by blocking $5 \mathrm{HT} 2$ receptors are associated substantially with obesity [6].

Amisulprid is a benzamide derivative that only binds to the D2/3 system [5] and is devoid of any significant af- finity to other receptor system, has a pronounced antipsychotic activity and lack of extrapiramidal side effects, even at high doses, when $90 \%$ of $\mathrm{D} 2$ receptors are blocked (Di Giovanini et al (1998) [7] demonstrated that chronic administration of amisupride produced a significant decrease of dopaminergic activity in the ventral midbrain region, the activity of dopamine neurons in nigro-striatal bundle being unchanged), produce a greater improvement in positive and negative symptom and has better outcome in longer term follow up studies compared to conventional serotonin-dopamine or multi-receptor atypical antipsychotics. Was demonstrated that it has a slow brain penetration. The increasing of prolactin level induced by amisulpride is lower compared to that produced by risperidone, olanzapine or haloperidol, and is not dependent on time [8]. It showed an interaction with the P-glycoprotein as a substrate. Amisulpride's main mode of entry is via the bloodcerebrospinal fluid barrier and this fact may alter not only its kinetics but also its brain distribution. Amisulpride have functional limbic selectivity in Fos expression like the other atypicals. Unlike other atypical antipsychotics, is not diabetogenic in man.

The results of the present study demonstrated a slightly weight gain after administration of amisulpride in both used doses $(1 \mathrm{mg} / \mathrm{kg}$ and $10 \mathrm{mg} / \mathrm{kg})$, with no difference regarding gender. The weight gain is lesser compared with other second generation antipsychotics (i.e. olanzapine and risperidone, widely used in therapy) [9]. In literature we found even data which claimed that that overweight patients treated with amisulpride showed significant weight loss [3].

Also, we recorded a slightly increase of blood glucose after treatment with amisulpride, the difference between initial and final blood sugar levels showed a statisticaly significant augmentation only at dose of $1 \mathrm{mg} / \mathrm{kg}$, but not at dose of $10 \mathrm{mg} / \mathrm{kg}$, so we consider that amisulpride in high dose does not influence the level of blood sugar, results confirmed by literature reference [2]. A study performed by Roix et al (2012) [10] which investigated the effects $(\mathrm{R} / \mathrm{S})$-amisulpride, and the chirally purified enantiomers, on glucose homeostasis in diet-induced obese (DIO) mice, demonstrated that amisulpride has antidiabetic actions in DIO mice resulting from increased insulin secretion and $\mathrm{R}$-amisulpride is antidiabetic and has minimal impact on prolactin levels. Kopf et al (2012) [8] demonstrated in a randomised, double blind cross-over design study on healthy volunteers, that a low dose of amisulpride, but not olanzapine appears to acutely increase pancreatic insulin secretion in healthy controls, and this stimulation of betacells could be a protective factor against the development of diabetes mellitus.

The assessement of cholesterol and triglycerides levels demonstrate obviously that amisulpride does not influence normal values even after a 9 weeks administration, at both used doses $(1 \mathrm{mg} / \mathrm{kg}$ and $10 \mathrm{mg} / \mathrm{kg})$, indicating that amisulpride does not produce lipid metabolism disturbances, 
which is a great advantage compared with atypical antipsychotics, olanzapine and risperidone [2,3]. In an openlabel 1-year study, Lin et al (2009) [11] investigated the effects on body weight and other metabolic profiles when psychiatric patients treated with another second generation antipsychotics were switched to amisulpride treatment and the results showed that the amisulpride-treated patients had significantly decreased fasting triglyceride, total cholesterol, glucose, and insulin resistance levels and a significant increase in high-density lipoprotein cholesterol levels after switching to amisulpride and the prevalence of metabolic syndrome in amisulpride-treated patients also decreased significantly. According to Peuskens et al (2007) [12] at doses that provide comparable control of psychosis, treatment with olanzapine was associated with greater increase in weight and blood glucose compared with amisulpride.

The explanation of this distinctive metabolic profile is supported by unique mechanism of action of amisulpride (in high doses showing affinity for $\mathrm{D} 2 / \mathrm{D} 3$ receptors, especially in mesolimbic and mesocortical system) $[7,8]$ and in low doses stimulating the dopaminergic transmission in the limbic system (at low doses blocks presynaptic D2/D3 receptors and in higer doses antagonize postsynaptic D2/ D3 receptor [13] and also showing a very low affinity or absence of binding for 5HT2 and 5HT7 receptors [8,13-15].

The assessement of serum transaminases showed that amisulpride did not increase the levels of SGOT and SGPT, lacking hepatotoxicity even for a long term administration, while in the literature there are many data reporting risperidone and olanzapine associated hepatotoxicity and cholestatic hepatitis [16-18].

In conclusion, amisulpride's high and selective affinity for dopamine D2/D3 receptors, lack of affinity for serotonin receptors, and its unusually high therapeutic doses makes it unique among atypical antipsychotics, showing that it has a favourable profile of metabolic and extrapiramidal side effects (in our experiments produced a slightly weight gain, in high dose the level of blood sugar, cholesterol and triglycerides levels were not influenced, indicating that amisulpride does not produce lipid metabolism disturbances and did not increase the levels of GOT and GPT, lacking hepatotoxicity even for a long term administration). All these benefits, briefly described in the literature, allow the acknowledge that amisulpride could be recommended to overweight or obese psychiatric patients or those which present a high risk of glucidic and lipidic metabolism disturbances.

\section{References}

1. Mortimer AM - Update on the management of symptoms in schizophrenia: focus on amisulpride. Neuropsychiatr Dis Treat. 2009;5:267-77

2. Gonçalves P, Araújo JR, Martel F - Antipsychotics-induced metabolic alterations: focus on adipose tissue and molecular mechanisms. Eur. Neuropsychopharmacol. 2015 Jan;25(1):1-16

3. Monreal Ortiz D, Chesa Vela E, Surribas Leza C, Franquelo C P.3.c.0671. The effects of amisulpride on weight and metabolic parameters in psychotic patients with surcharge weight, European Neuropsychopharmacology, 2009, 19 (9): S548-S549

4. Patsch JR, Ebenbichler CF - Metabolic side effects of antipsychotic medication. International Journal Clinical Practice 2007, 61, 8, 13561370

5. Correll CU, Lencz T, Malhotra AK - Antipsychotic drugs and obesity. Trends Mol Med. 2011 Feb;17(2):97-107.

6. Reynolds GP, Kirk SL - Metabolic side effects of antipsychotic drug treatment--pharmacological mechanisms.Pharmacol Ther. 2010 Jan;125(1):169-79

7. Di Giovanni G, Di Mascio M, Di Matteo $\vee$ et al - Effects of acute and repeated administration of amisulpride, a dopamine D2/D3 receptor antagonist, on the electrical activity of midbrain dopaminergic neurons. J Pharmacol Exp Ther. 1998 Oct;287(1):51-57.

8. Natesan S, Reckless GE, Barlow KBL - Amisulpride-the 'atypical' atypical antipsychotic-Comparison to haloperidol, risperidone and olanzapine.Schizophrenia Research, 2008, 105:224-235

9. Vanelle JM, Douki S - A double-blind randomised comparative trial of amisulpride versus olanzapine for 2 months in the treatment of subjects with schizophrenia and comorbid depression.Eur Psychiatry.2006;21(8):523-30

10. Roix JJ, DeCrescenzo GA, Cheung PH et al - Effect of the antipsychotic agent amisulpride on glucose lowering and insulin secretion.Diabetes Obes Metab. 2012 Apr;14(4):329-34

11. Lin CC, Bai YM, Wang YC et al - Improved body weight and metabolic outcomes in overweight or obese psychiatric patients switched to amisulpride from other atypical antipsychotics.J Clin Psychopharmacol. 2009 Dec;29(6):529-36

12. Peuskens J. De Hert M, Mortimer A - SOLIANOL Study Group. Metabolic control in patients with schizophrenia treated with amisulpride or olanzapine. Int Clin Psychopharmacol. 2007 May;22(3):145-52

13. Schoemaker $\mathrm{H}$, Claustre $\mathrm{Y}$, Fage $D$ et al - Neurochemical characteristics of amisulpride, an atypical dopamine D2/D3 receptor antagonist with bothpresynaptic and limbic selectivity.J Pharmacol Exp Ther. 1997; 280(1):83-97

14. Donahue TJ, Hillhouse TM, Webster KA et al - (S)-amisulpride as a discriminative stimulus in C57BL/6 mice and its comparison to the stimulus effects of typical and atypical antipsychotics. Eur J Pharmacol. 2014 Jul 5;734:15-22

15. Perrault G, Depoortere R, Morel E et al - Psychopharmacological profile of amisulpride: an antipsychotic drug with presynaptic D2/D3 dopamine receptorantagonist activity and limbic selectivity.J Pharmacol Exp Ther. $1997 ; 280(1): 73-82$

16. Krebs S, Dormann H, Muth-Selbach $U$ et al - Risperidone-induced cholestatic hepatitis.Eur J Gastroenterol Hepatol. 2001 Jan;13(1):67-9

17. López-Torres E, Süveges A, Peñas-LLedó EM et al - Liver enzyme abnormalities during antipsychotic treatment: a case report of risperidone-associated hepatotoxicity.Drug Metabol Drug Interact. 2014;29(2):123-6

18. Manceaux P, Constant E, Zdanowicz N et al - Management of marked liver enzyme increase during olanzapine treatment: a case report and review of the literature.Psychiatr Danub. 2011 Sep;23 Suppl 1:S15-7. 